\title{
Efecto de aceites esenciales de orégano en la calidad de la carne de codornices
}

\section{Effect of oregano essential oils on meat quality of quail}

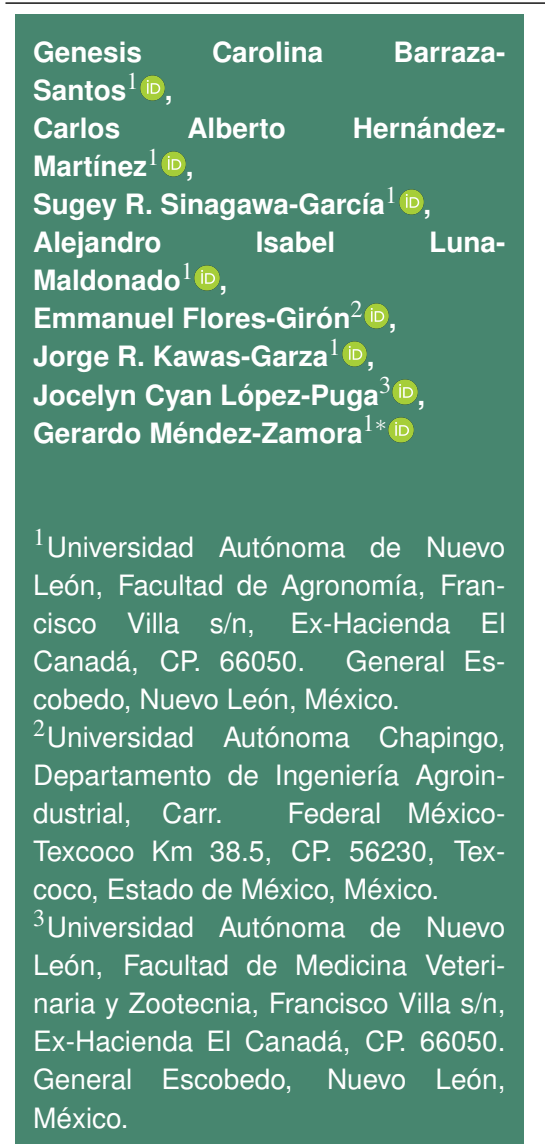

*Autor de correspondencia:

gerardo.mendezzm@uanl.edu.mx;

mezage@hotmail.com

\section{Nota científica}

Recibida: 26 de agosto 2020

Aceptada: 10 de mayo 2021
Como citar: Barraza-Santos GC Hernández-Martínez CA, SinagawaGarcía SR, Luna-Maldonado Al, Flores-Girón E, Kawas-Garza JR, López-Puga JC, Méndez-Zamora G (2021) Efecto de aceites esenciales de orégano en la calidad de la carne de codornices. Ecosistemas y Recursos Agropecuarios 8(2): e2709. DOI: 10.19136/era.a8n2.2709
RESUMEN. Este estudio evaluó la calidad de la carne de codorniz suplementadas con aceites de orégano (AEO) Lippia berlandieri Schauer (Lb) y Poliomintha longiflora Gray(PI). Los tratamientos fueron CON: dieta control, Lb: $0.20 \mathrm{~g} \mathrm{~kg}^{-1}$ Lippia berlandieri Schauer en dieta y Pl: $0.20 \mathrm{~g} \mathrm{~kg}^{-1} P O-$ liomintha longiflora Gray en dieta. Las variables de sacrificio, fisicoquímicas, textura y sensoriales fueron evaluadas. El peso sacrificio incrementó $(P<$ $0.05)$ en $\mathrm{Lb}$. El pH y capacidad de retención de agua (CRA) disminuyeron $(\mathrm{P}<0.05)$ en $\mathrm{Lb}$ y $\mathrm{Pl}$, mientras que CON presentó $(\mathrm{P}<0.05)$ más CRA. En PI se obtuvo el mejor valor $(P<0.05)$ de luminosidad. La cohesividad y resistencia de la carne incrementaron $(P<0.05)$ para CON; sin embargo, los atributos sensoriales no cambiaron $(P>0.05)$. En conclusión, los aceites de orégano en $0.20 \mathrm{~g} \mathrm{~kg}^{-1}$ mejoran los rendimientos sacrificio y calidad de la carne.

Palabras clave: Atributos; calidad; color; sensorial; textura.

ABSTRACT. This study was to evaluate meat quality of quails supplemented with oregano essential oils (OEO) Lippia berlandieri Schauer (Lb) and Poliomintha longiflora Gray (PI). Experimental diets consisted on CON: control diet, Lb: $0.20 \mathrm{~g} \mathrm{~kg}^{-1}$ Lippia berlandieri Schauer and Pl: $0.20 \mathrm{~g}$ $\mathrm{kg}^{-1}$ Poliomintha longiflora Gray added on diet. Variables of slaughter, physicochemicals, texture and sensorial attributes were evaluated. $\mathrm{Lb}$ increased $(P<0.05)$ slaughter weight. The $\mathrm{pH}$ and water holding capacity $(\mathrm{WHC})$ decreased $(\mathrm{P}<0.05)$ for $\mathrm{Lb}$ and $\mathrm{PI}$ treatments, while CON treatment showed higher $(P<0.05)$ WHC. $P I$ had the best value on lightness $(P<0.05)$. CON increased $(P<0.05)$ cohesiveness and resilience of meat; however, sensory atributtes did not change $(P>0.05)$. In conclusion, $0.20 \mathrm{~g} \mathrm{~kg}^{-1}$ of OEO in diet improved slaughter yields and meat quality.

Key words: Attributes; quality; color; sensory; texture. 


\section{INTRODUCCIÓN}

La tasa de crecimiento y eficiencia alimenticia son las principales características de la producción avícola, considerando el potencial genético, calidad de las dietas, condiciones ambientales y brotes de enfermedades como factores para el óptimo desarrollo de las aves (Sugiharto 2016). Características que influyen en la producción de carne y su calidad en $\mathrm{pH}$, color, textura y propiedades sensoriales. En este sentido, los antibióticos y su efectividad en la producción de aves han resultado efectivos; sin embargo, la conciencia pública y el conocimiento sobre los riesgos potenciales para la salud por los promotores de crecimiento y la demanda de alimentos orgánicos han cambiado la perspectiva del consumidor. Por lo que la tendencia de encontrar alternativas naturales ha aumentado. Una de estas ha sido el uso de extractos vegetales de plantas aromáticas como los aceites esenciales de romero, tomillo y orégano. En este sentido, el aceite esencial de orégano (AEO) ha sido más investigado en los pollos de engorda (MéndezZamora et al. 2015ab, Cázares-Gallegos et al. 2019, Hernández-Coronado et al. 2019, Sánchez-Zamora et al. 2019) que en otras aves como los pavos y codornices (Sadi Cetingul et al. 2009, Yesilbag et al. 2012).

En codornices, las investigaciones realizadas sobre el uso de orégano (Oregano onites) han sido sobre el comportamiento productivo y calidad de huevo (Sadi Cetingul et al. 2009), siendo $50 \mathrm{~g} \mathrm{~kg}^{-1}$ una dosis apropiada. Otros estudios en codornices involucran la combinación de aceite de orégano con el de romero (Yesilbag et al. 2012), timol (Lábaque et al. 2013), canela (Mehdipour et al. 2013), Mentha spicata (Ghazaghi et al. 2014), menta (Mehri et al. 2015) y aceites esenciales de hierbas (Hajiaghapour y Rezaeipour 2018); adicionalmente también el aceite de enebro (Yesilbag et al. 2014), suplementación de canola (Mnisi y Mlambo 2018), y otros ingredientes como la moringa en polvo (Castillo et al. 2018), Pleurotus ostreatus (Vargas-Sánchez et al. 2018) y calostro en polvo (Fatih Akdemir et al. 2018). Estos estudios han evaluado estos suplementos en el com- portamiento productivo de las codornices y han indicado que los ingredientes naturales pueden usarse en las dietas para mejorar la producción y calidad de la carne de las codornices. Es así como el AEO podría ser una alternativa en las dietas de las codornices para mejorar la producción de carne y calidad de la carne, ya que en pollos de engorda ha mejorado la productividad y calidad de la carne (MéndezZamora et al. 2015ab, Cázares-Gallegos et al. 2019, Hernández-Coronado et al. 2019, Sánchez-Zamora et al. 2019).

Relacionado con los AEO, estudios son necesarios para evaluar el $\mathrm{pH}$, retención de agua, color, textura y atributos sensoriales de la carne de codornices; entre estas opciones está el AEO Mexicano que incluyen los AEO Lippia berlandieri Schauer (Lb) y Poliomintha longiflora Gray (PI). Estos AEO no han sido reportados en la producción de codornices ni en los efectos del rendimiento de carne y su calidad. Por lo que el objetivo de este estudio fue evaluar el efecto de aceites esenciales de orégano Lb y PI sobre variables de canal, propiedades fisicoquímicas, textura y aceptación sensorial de la carne de codornices.

\section{MATERIALES Y MÉTODOS}

La producción y engorda de codornices fue hecha en el Campus La Ascensión de la Facultad de Agronomía, Universidad Autónoma de Nuevo León, en Aramberri, N.L., México. La Ascensión está localizada en latitud $26^{\circ} 49^{\prime} \mathrm{N}$, longitud $-100^{\circ} 19^{\prime}$ $\mathrm{O}$ y altitud $500 \mathrm{msnm}$, con un clima predominante semiseco, precipitación media anual de $535 \mathrm{~mm}$, temperatura media de $13.95{ }^{\circ} \mathrm{C}$ y humedad relativa promedio es de $67 \%$ (INEGI 2020).

Un total de 75 codornices se muestrearon de un experimento completamente al azar de tres tratamientos, donde recibieron $(200 \mathrm{ppm}=0.20 \mathrm{~g}$ $\mathrm{kg}^{-1}$ ) aceites de orégano Lippia berlandieri Schauer (Lb) y Poliomintha longiflora Gray (PI) en las dietas; CON: dieta control, Lb: $0.20 \mathrm{~g} \mathrm{~kg}^{-1}$ Lippia berlandieri Schauer en dieta y PI: $0.20 \mathrm{~g} \mathrm{~kg}^{-1}$ Poliomintha longiflora Gray en dieta. Un total de 5 codornices de las cinco jaulas por tratamiento fueron seleccionados 
aleatoriamente al final del estudio ( $\mathrm{n}=25$ codornices/tratamiento) para la evaluación. Los AEO fueron adquiridos de la empresa Natural Solutions S.M.I. (Cd. Jiménez, Chihuahua, México), obtenidos por arrastre de vapor y la composición fue como sigue: Lb $60.00 \%$ carvacrol y $3.91 \%$ timol, y $\mathrm{PI}$ $13.80 \%$ carvacrol y $28.40 \%$ timol, reportados por Hernández-Coronado et al. (2019). La formulación de las dietas se muestra en la Tabla 1 de acuerdo con la NRC (1994) para la fase iniciación (1-21 d) y finalización (22-55 d). El AEO fue mezclado con el aceite vegetal de la dieta para su incorporación. La alimentación de las aves fue ad libitum de alimento y agua, con una temperatura de $36^{\circ} \mathrm{C}$ en la primer semana y después disminuida $4{ }^{\circ} \mathrm{C}$ por semana y terminar a los 21 días con $28^{\circ} \mathrm{C}$, y así se mantuvo hasta el cierre del experimento a los 55 días.

Tabla 1. Ingredientes de las dietas formuladas para las codornices.

\begin{tabular}{lcc}
\hline \multirow{2}{*}{ Ingredientes $\left(\mathrm{g} \mathrm{kg}^{-1}\right)$} & \multicolumn{2}{c}{ Dietas } \\
\cline { 2 - 3 } & $1-21 \mathrm{~d}$ & $22-55 \mathrm{~d}$ \\
\hline Sorgo & 90.0 & 130.0 \\
Harina de soya & 650.0 & 570.0 \\
Maíz molido & 100.0 & 140.0 \\
Aceite vegetal & 8.0 & 8.0 \\
Carbonato de calcio & 52.0 & 52.0 \\
Vitaminas y minerales & 100.0 & 100.0 \\
\hline
\end{tabular}

A los 55 día de acuerdo con la Norma Oficial Mexicana NOM-033-ZOO-2014 (SE 2014) se realizó el sacrificio ( $n=25$ codornices). El peso al sacrificio (PS), peso de canal caliente (PCC) y fría (PCF) $24 \mathrm{~h}$ post mortem fueron registrados y el rendimiento de la canal caliente (RCC; (PCC/PS) x 100) y fría (RCF; (PCF/PS) x 100) fueron obtenidos. Además, el rendimiento de piezas (pechuga, muslo, pierna, alas, cadera y espalda (CyE)) fueron estimados pesando cada pieza y calculando su rendimiento en función del PS ((Peso pieza/PS) x 100).

$\mathrm{El} \mathrm{pH}$, color, capacidad de retención de agua (CRA) y pérdida por cocción (PPC) de la pechuga cruda de codorniz se determinaron $24 \mathrm{~h}$ post mortem. Estas variables se midieron por duplicado en ocho pechugas de cada tratamiento, seleccionadas completamente al azar. El pH de la pechuga fue realizado con un potenciómetro (Orion 3 star ThermoFisher
Scientific, Pittsburgh, PA, U.S.A.) mediante un electrodo de punción. Con un colorímetro (CR-400 Konica Minolta $\AA$, Tokyo, Japón) se determinaron las variables luminosidad $\left(\mathrm{L}^{*}\right)$, tendencia al color rojo $\left(a^{*}\right)$, tendencia al amarillo $\left(b^{*}\right)$, Chroma (índice de saturación) y ángulo Hue (tono) sobre la superficie externa de la pechuga en las ocho muestras por tratamiento. $L^{*}, a^{*} y b^{*}$ fueron usados para determinar el cambio de color total $(\Delta \mathrm{CT})$ y el índice de coloración (IC) como lo realizaron HernándezCoronado et al. (2019) y Cázares-Gallegos et al. (2019). La capacidad de retención de agua (CRA) de cada pechuga fue obtenida de acuerdo con la técnica descrita por Cázares-Gallegos et al. (2019), registrando el peso inicial $(\mathrm{Pi})$ y final $(\mathrm{Pf})$ de la muestra $(C R A=100-[((P i-P f) / P i) \times 100])$. Para la PPC, la carne de la pechuga deshuesada fue empacada (Koch 800, Kansas City, MO) en bolsas de vacío (Zubex Industrial SA de CV, Monterrey, Nuevo León, México) y cocinada a $75 \pm 0.1{ }^{\circ} \mathrm{C}$ durante $1 \mathrm{~h} 30 \mathrm{~min}$ por inmersión en agua caliente. Después las muestras fueron enfriadas por baño en agua en $4{ }^{\circ} \mathrm{C}$ por 20 min, removidas de las bolsas y escurriéndolas cuidadosamente. Los pesos de la carne cruda y cocida se registraron para evaluar esta variable $[\%$ PPC = ((peso crudo - peso cocido) / peso crudo) x 100].

En el análisis de textura de la carne, la preparación de las muestras fue similar al método para evaluar la pérdida por cocción (carne empacada al vacío, cocido $1 \mathrm{~h} 30 \mathrm{~min}$ a $75^{\circ} \mathrm{C}$ ), pero después del cocimiento y pre enfriado $\left(4{ }^{\circ} \mathrm{C} / 20 \mathrm{~min}\right)$, las muestras se almacenaron en refrigeración $\left(4^{\circ} \mathrm{C}\right)$ por $12 \mathrm{~h}$. La fuerza de corte (FC) y el análisis de perfil de textura (APT) se realizaron en nueve pechugas de cada tratamiento muestreando por cada lado para FC (gf) y APT ( $n=18 ; 2$ pechuga/tratamiento), y realizar su análisis a $4{ }^{\circ} \mathrm{C}$ con un analizador de textura (TA.XT.Plus, Stable Micro Systems Serrey, England). En FC se utilizaron piezas rectangulares de $0.5 \mathrm{~cm}$ ancho $\times 0.5 \mathrm{~cm}$ alto $\times 2.0 \mathrm{~cm}$ largo de la carne cocida. En APT se usaron cilindros de carne obtenidos con un sacabocados, estandarizados a $1.0 \mathrm{~cm}$ de diámetro y $0.7 \mathrm{~cm}$ de altura. Las variables del APT obtenidas fueron dureza ( $g f)$, elasticidad (mm), cohesividad (adimensional), gomosidad 
(g), masticabilidad ( $\mathrm{g} \mathrm{mm}$ ), resistencia (adimensional) y adhesividad $\left(\mathrm{g} \mathrm{s}^{-1}\right)$. Las condiciones de prueba en el texturómetro se establecieron de acuerdo con lo reportado por Sánchez-Zamora et al. (2019).

Una prueba sensorial afectiva por atributos se efectuó para determinar el grado de satisfacción de 30 consumidores, basados en los estudios realizados por Hernández-Coronado et al. (2019) y CázaresGallegos et al. (2019). Para esta evaluación, ocho pechugas al azar por tratamiento fueron deshuesadas, empacadas al vacío y cocinadas a $75{ }^{\circ} \mathrm{C}$ por $1 \mathrm{~h} 30 \mathrm{~min}$ y enfriadas a $4{ }^{\circ} \mathrm{C}$ en agua con hielo y almacenadas a $4{ }^{\circ} \mathrm{C}$ durante $12 \mathrm{~h}$ hasta su evaluación. Cada consumidor recibió cuatro cubos de $1.0 \mathrm{~cm}$ por lado a una temperatura mantenida a 28 ${ }^{\circ} \mathrm{C}$, colocados en vasos de plástico codificados con tres números al azar. Los consumidores seleccionados fueron aquellos que consumen carne de manera cotidiana y entre una edad de 25 a 34 años. Los atributos para evaluar fueron color, olor, sabor, suavidad y aceptabilidad global. En esta prueba fue usada una escala hedónica de siete puntos, donde $7=$ me gusta mucho y $1=$ me disgusta mucho.

Las variables de sacrificio y calidad de la carne fueron evaluadas con un análisis de varianza, usando el modelo lineal general de Minitab (versión 17.3.0, 2014) y el siguiente modelo estadístico: $\mathrm{y}_{i j}=\mu+$ $\tau_{i}+\varepsilon_{i j}$; dónde: $y_{i j}=$ variables de sacrificio, despiece, fisicoquímicas, textura y sensorial; $\mu=$ media general; $\tau_{i}=$ efecto del i-esimo tratamiento; $\varepsilon_{i j k}=$ error aleatorio distribuido en forma normal con media y varianza $\left[\varepsilon_{i j} \sim \mathrm{N}\left(\mu, \sigma^{2}\right)\right]$. En el caso del análisis sensorial el modelo estadístico incluyó el efecto del bloque, el cual el evaluador fue considerado como este término. Cuando existió rechazo de hipótesis nula (H0; P-value < 0.05), la comparación de medias fue realizada con la instrucción Tukey.

\section{RESULTADOS Y DISCUSIÓN}

El efecto del aceite esencial de orégano mexicano (AEO) sobre las variables de sacrificio está presente en la Tabla 2. El PS fue afectado $(P<0.05)$ por los niveles de $0.20 \mathrm{~g} \mathrm{~kg}^{-1}$ AEO entre los tratamientos evaluados, donde Lb obtuvo el mejor peso y CON el valor más bajo. También, 0.10 y $0.14 \mathrm{~g} \mathrm{~kg}^{-1}$ de AEO en dietas de codornices incrementaron el PS (Yesilbag et al. 2012). Los valores presentados en PS contrastan con los obtenidos por Vargas-Sánchez et al. (2018) cuando evaluaron 0,10 y $20 \mathrm{~g} \mathrm{~kg}^{-1}$ de $P$. ostreatus en agua de beber y en dieta, quiénes obtuvieron mayores PS y menores rendimientos de canal sin diferencias entre sus grupos experimentales. Este contraste puede deberse a la diferencia en composición y estructura química entre los AEO y $P$. ostreatus. Por otra parte, en RCC y RFC no hubo diferencia estadística $(P>0.05)$ entre tratamientos. No obstante, las canales del grupo control (CON) presentaron la media alta. Mnisi y Mlambo et al. (2018) reemplazaron 2.5, 5.0, 12.5 y $17.5 \%$ de harina de canola (Brassica napus) por harina de soya (Glycine max) en dietas, los resultados obtenidos presentaron un comportamiento similar a los evaluados en este estudio en las variables RCC y RCF; esto indica que $0.20 \mathrm{~g}$ $\mathrm{kg}^{-1}$ de AEO en las dietas de codornices tiene un efecto similar a la harina de soya en el rendimiento de las piezas de la canal. Las diferencias en las variables de sacrificio pueden deberse al tipo y nivel de AEO (Yesilbag et al. 2012, Hernández-Coronado et al. 2019). En pollos de engorda, $0.20 \mathrm{~g} \mathrm{~kg}^{-1}$ de AEO $\mathrm{Lb}$ en dieta incrementaron el PS, relacionado con el efecto del AEO como promotor de crecimiento, mejorador de la digestibilidad y absorción de los nutrientes (Sánchez-Zamora et al. 2019). Esta aseveración puede explicar como el AEO de Lb y PI en $0.20 \mathrm{~g}$ $\mathrm{kg}^{-1}$ puede ser un promotor de crecimiento, ya que obtuvieron los mayores PS.

Tabla 2. Variables de sacrificio de codornices suplementadas con aceites de orégano.

\begin{tabular}{|c|c|c|c|}
\hline \multirow{2}{*}{ Tratamientos } & \multicolumn{3}{|c|}{ Variables de sacrificio } \\
\hline & PS (g) & $\mathrm{RCC}(\%)$ & RCF (\%) \\
\hline $\mathrm{CON}$ & $138.52^{b}$ & $66.39^{a}$ & $63.70^{a}$ \\
\hline $\mathrm{Lb}$ & $152.24^{a}$ & $64.02^{a}$ & $61.16^{a}$ \\
\hline $\mathrm{Pl}$ & $149.56^{a b}$ & $64.11^{a}$ & $60.83^{a}$ \\
\hline EE & 3.31 & 1.09 & 1.05 \\
\hline$P$ value & 0.014 & 0.232 & 0.115 \\
\hline \multicolumn{4}{|c|}{$\begin{array}{l}\mathrm{CON}=\text { dieta control; } \mathrm{Lb}=0.20 \mathrm{~g} \mathrm{~kg}^{-1} \text { Lippia } \\
\text { berlandieri Schauer en dieta; } \mathrm{PI}=0.20 \mathrm{~g} \mathrm{~kg}^{1} P o- \\
\text { liomintha longiflora Gray en dieta; } \mathrm{EE}=\text { error están- } \\
\text { dar. } \mathrm{PS}=\text { peso sacrificio; } \mathrm{RCC}=\text { rendimiento canal } \\
\text { caliente; RCF = rendimiento canal fría. }{ }^{a-b} \text { Medias ( } \mathrm{n} \\
=25 \text { por tratamiento) en columnas y con diferente su- } \\
\text { períndice son diferentes significativamente }(\mathrm{P}<0.05) \text {. }\end{array}$} \\
\hline
\end{tabular}


En términos generales, no se mostraron diferencias estadísticas $(P>0.05)$ entre los tratamientos evaluados para las variables de despiece de codornices suplementadas con aceite de orégano (Tabla 3). Para la variable pechuga, el tratamiento Lb presentó los valores altos, pero CON superó en las variables restantes. En comparación con investigaciones previas, Ghazaghi et al. (2014) evaluaron 0 , $1,2,3$ y $4 \%$ de Mentha spicata en la dieta y no encontraron diferencia en pechuga y muslo; en adición 200, 300 y $400 \mathrm{mg} \mathrm{kg}^{-1}$ de aceite esencial de Thymus vulgaris, Satureja hortensis y Mentha pulegium no afectan el rendimiento canal, pechuga y muslo de codorniz (Naghmeh Dehghani et al. 2018). Adicionalmente, Mnisi y Mlambo et al. (2018) no obtuvieron efectos en las alas. Así mismo, Sánchez-Zamora et al. (2019) no encontraron diferencias en las piezas, excepto en alas de la canal de pollos de engorda suplementados con $0.20 \mathrm{~g} \mathrm{~kg}^{-1}$ AEO; esto puede ser debido a que de $0.20 \mathrm{~g} \mathrm{~kg}^{-1}$ AEO en dieta no es un nivel de inclusión que pueda tener efecto sobre la división celular de las miofibrillas (Sánchez-Zamora et al. 2019), y en consecuencia el despiece de las codornices no resultó afectado por el AEO en las dietas de las codornices.

Tabla 3. Despiece de las canales de codornices suplementadas con aceites de orégano.

\begin{tabular}{cccccc}
\hline \multirow{2}{*}{ Tratamientos } & \multicolumn{5}{c}{ Variables de despiece } \\
\cline { 2 - 6 } & Pechuga & Muslo & Pierna & Alas & CyE \\
\hline CON & $24.51^{a}$ & $9.49^{a}$ & $5.91^{a}$ & $5.14^{a}$ & $19.11^{a}$ \\
$\mathrm{Lb}$ & $24.56^{a}$ & $9.40^{a}$ & $5.85^{a}$ & $4.92^{a}$ & $18.22^{a}$ \\
$\mathrm{Pl}$ & $23.24^{a}$ & $8.95^{a}$ & $5.57^{a}$ & $4.65^{a}$ & $17.77^{a}$ \\
$\mathrm{EE}$ & 0.47 & 0.18 & 0.16 & 0.17 & 0.52 \\
$\mathrm{P}$ value & 0.091 & 0.080 & 0.316 & 0.151 & 0.190 \\
\hline
\end{tabular}

$\mathrm{CON}=$ dieta control; $\mathrm{Lb}=0.20 \mathrm{~g} \mathrm{~kg}^{-1}$ Lippia berlandieri Schauer en dieta; $\mathrm{PI}=0.20 \mathrm{~g} \mathrm{~kg}^{-1}$ Poliomintha longiflora Gray en dieta; $\mathrm{EE}=$ error estándar. CyE = cadera y espalda. ${ }^{a}$ Medias ( $\mathrm{n}=25$ por tratamiento) en columnas y con diferente superíndice son diferentes significativamente $(P<0.05)$.

El pH y CRA presentaron diferencia estadística $(P<0.05)$ entre tratamientos (Tabla 4). El pH decreció en los tratamientos con AEO (Lb y PI); esto indicó que al suplementar estos aceites en la dieta puede disminuir el pH de la carne. Así mismo, la CRA de los tratamientos se comportaron parecidos, ya que $\mathrm{Lb}$ disminuyó CRA, mientras que en CON aumentó. La
PPC fue similar $(P>0.05)$ entre los grupos experimentales. Mentha piperita en 20,30 y $40 \mathrm{~g} \mathrm{~kg}^{-1}$ no afectan el $\mathrm{pH}$ y CRA de la carne de codorniz en cinco semanas de experimentación (Mehri et al. 2015). Dosis de 100 y $200 \mathrm{mg}$ de aceite de canela y 1 y $2 \mathrm{~g}$ de canela en polvo no influyeron en el $\mathrm{pH}$ de la carne de codorniz (Mehdipour et al. 2013); pero estas dosis si influyeron en CRA con valores superiores a los del presente estudio usando $0.20 \mathrm{~g} \mathrm{~kg}^{-1}$ de AEO Lb y Pl. En el caso del pH, su valor bajo puede deberse a que los compuestos antioxidantes reactivos con lípidos y radicales hidroxilos (Mehdipour et al. 2013), se acumulen en la carne después del rigor mortis, cediendo grupos hidroxilos en el medio y haga que el pH disminuya. Vargas-Sánchez et al. (2018) también obtuvieron efectos en $\mathrm{pH}$ y CRA, pero con valores más bajos en $\mathrm{pH}$ y mayores en CRA (2\%). Estos resultados con $0.20 \mathrm{~g} \mathrm{~kg}^{-1}$ de Lb y PI pueden afectar las características fisicoquímicas de la carne de codorniz, ya que las moléculas activas (timol y carvacrol) del AEO afectan el comportamiento productivo. Los resultados obtenidos en CRA indicaron que disminuye a $\mathrm{pH}$ menores a 6.0 , en este caso $\mathrm{Lb}$ y $\mathrm{PI}$ obtuvieron los menores $\mathrm{pH}$. Aun así, la CRA no puede considerarse afectada por el $\mathrm{pH}$, ya que éste no está cerca al $\mathrm{pH}$ del punto isoeléctrico de la miosina (5.4), que podría retraer las miofibrillas y por lo tanto afectar la CRA (Sánchez-Zamora et al. 2019). En pollos de engorda, niveles ascendentes de 0.2 a $1.0 \mathrm{~g} \mathrm{~kg}^{-1}$ de AEO Lb en dietas disminuyeron el $\mathrm{pH}(5.72-5.84)$ de la carne, pero estos valores no afectaron la CRA (56.67-60.32) aunque esta variable puede incrementar a niveles altos de AEO (CázaresGallegos et al. 2019). En el presente estudio con 0.20 $\mathrm{g} \mathrm{kg}^{-1}$ de AEO Lb y PI en dietas de las codornices fueron mayores a los obtenidos en pollos de engorda (Cázares-Gallegos et al. 2019). Con estos contrastes puede indicarse que a este nivel no se reduce el $\mathrm{pH}$ de la carne de codornices.

En la Tabla 5 se puede observar que la $L^{*}$ y el cambio de color total $(\Delta \mathrm{CT})$ en la pechuga de codorniz hubo diferencia $(P<0.05)$ entre los tratamientos. El tratamiento PI obtuvo la mayor $L^{*}$, implicando que CON presente la menor, pero incrementara el $\Delta \mathrm{CT}$. En cuanto a $a^{*}, b^{*}$, Hue, Chroma e IC no mostraron 
diferencias estadísticas $(P>0.05)$ entre los tratamientos. Los valores obtenidos en $L^{*}$ y $\Delta C T$ por CázaresGallegos et al. (2019) con la adición de diferentes concentraciones de AEO en las dietas (200, 400, 600, 800 y $1000 \mathrm{mg} \mathrm{kg}^{-1}$ ) de pollos de engorda superaron a los presentados en carne de codorniz con similar tendencia a incrementar en los tratamientos con AEO.

Tabla 4. pH, capacidad de retención de agua y pérdida por cocción en las pechugas de codorniz suplementadas con aceites de orégano.

\begin{tabular}{|c|c|c|c|}
\hline \multirow{2}{*}{ Tratamientos } & \multicolumn{3}{|c|}{ Variables fisicoquímicas } \\
\hline & $\mathrm{pH}$ & CRA (\%) & PPC (\%) \\
\hline CON & $6.04^{a}$ & $62.74^{a}$ & $38.25^{a}$ \\
\hline Lb & $5.96^{a b}$ & $58.16^{b}$ & $39.10^{a}$ \\
\hline $\mathrm{PI}$ & $5.94^{b}$ & $60.34^{a b}$ & $39.06^{a}$ \\
\hline EE & 0.03 & 0.71 & 0.73 \\
\hline$P$ value & 0.021 & 0.000 & 0.6740 \\
\hline \multicolumn{4}{|c|}{$\begin{array}{l}\mathrm{CON}=\text { dieta control; } \mathrm{Lb}=0.20 \mathrm{~g} \mathrm{~kg}^{-1} \text { Lippia } \\
\text { berlandieri Schauer en dieta; } \mathrm{PI}=0.20 \mathrm{~g} \mathrm{~kg}^{-1} \text { Po- } \\
\text { liomintha longiflora Gray en dieta; } \mathrm{EE}=\text { error están- } \\
\text { dar. } \mathrm{pH}=\text { potencial de hidrógeno; } \mathrm{CRA}=\text { capacidad } \\
\text { de retención de agua; } \mathrm{PPC}=\text { pérdida por cocido. } \\
a-b \text { Medias }(\mathrm{n}=8 \text { por tratamiento) en columnas y } \\
\text { con diferente superíndice son diferentes significati- } \\
\text { vamente }(\mathrm{P}<0.05) \text {. }\end{array}$} \\
\hline
\end{tabular}

En las variables de textura de las pechugas de codornices suplementadas con aceite de orégano, únicamente la cohesividad y resistencia obtuvieron diferencia significativa $(P<0.05)$ entre los tratamientos (Tabla 6). De hecho, CON presentó los mayores valores en estas variables. En relación con cohesividad y resistencia, similares efectos estadísticos presentaron las investigaciones de Cázares-Gallegos et al. (2019) y Hernández-Coronado et al. (2019) cuando evaluaron $200-1000 \mathrm{mg} \mathrm{kg}^{-1}$ y $400 \mathrm{mg} \mathrm{kg}^{-1}$ $\mathrm{Lb}$ y $\mathrm{PI}$ en pollos de engorda. Además, similarmente a los estudios antes mencionados, el comportamiento de los resultados en estas variables decreció en comparación con el tratamiento CON. Estas comparaciones indicaron que las aves que consumieron $A E O$ durante su producción, influyen en las propiedades texturales de la carne ya que el AEO puede causar un efecto restrictivo en el metabolismo ante mortem y previene un mayor daño de las proteínas miofibrilares y estructura de la carne de pechuga (Hernández-Co- ronado et al. 2019).

En términos generales para la evaluación sensorial de la pechuga de codorniz suplementadas con AEO en la dieta (Tabla 7), la preferencia de color, olor, sabor, suavidad y aceptabilidad global de la carne de codorniz no fue diferente $(P>0.05)$ entre los tratamientos evaluados de las dietas que consumieron las codornices. De manera global, los consumidores prefirieron el tratamiento $\mathrm{Lb}$, ya que este obtuvo las puntuaciones altas de preferencia. Aunque no se encontró diferencia en los atributos sensoriales de la carne de codorniz de los diferentes tratamientos, con los resultados obtenidos en este estudio puede indicarse que el tratamiento $L b$ fue el mejor aceptado sensorialmente, a excepción de olor donde $\mathrm{Pl}$ fue el mejor aceptado. En pollos de engorda suplementados con AEO Lb (200-1000 mg $\mathrm{kg}^{-1}$ ) en dieta no influyeron el olor, sabor, suavidad y aceptabilidad global (Cázares-Gallegos et al. 2019), pero numéricamente la carne de pechuga fue más aceptada, así como sucedió en la preferencia de la carne de codorniz. Diferencias pueden ser encontradas si el AEO es suplementado en el agua de bebida, así como lo obtuvieron Hernández-Coronado et al. (2019) en el olor, sabor y aceptabilidad general de la carne de pechugas de pollo suplementados con AEO Lb y $\mathrm{PI}\left(400 \mathrm{mg} \mathrm{kg}^{-1}\right)$.

El uso de los aceites de orégano (AEO) Poliomintha longiflora Gray (PI) y Lippia berlandieri Schauer (Lb) en $0.20 \mathrm{~g} \mathrm{~kg}^{-1}$ en dieta, pueden considerarse como aditivos benéficos sobre el rendimiento en variables de sacrificio y despiece de la canal, así como en calidad de la carne. Destacando Lb por su influencia en las características de sacrificio y despiece, así como contribución al mejorar la fuerza de corte y disminuir la pérdida por cocción. Además, los AEO aumentaron la luminosidad y disminuyeron el pH, retención de agua, cambio de color, cohesividad y resistencia de la carne. Los aceites esenciales de orégano incrementaron la aceptabilidad sensorial de los atributos de la carne de codorniz. 
Tabla 5. Efecto del aceite de orégano en dietas sobre los parámetros de color en pechuga de codorniz.

\begin{tabular}{|c|c|c|c|c|c|c|c|}
\hline \multirow{2}{*}{ Tratamientos } & \multicolumn{7}{|c|}{ Variables color } \\
\hline & $L^{*}$ & $a^{*}$ & $\mathrm{~b}^{*}$ & Hue & Chroma & $\Delta \mathrm{CT}$ & IC \\
\hline CON & $46.02^{b}$ & $7.08^{a}$ & $3.75^{a}$ & $29.61^{a}$ & $8.19^{a}$ & $48.90^{a}$ & $19.40^{a}$ \\
\hline $\mathrm{Lb}$ & $48.55^{a}$ & $6.36^{a}$ & $4.25^{a}$ & $33.46^{a}$ & $7.87^{a}$ & $46.27^{b}$ & $18.57^{a}$ \\
\hline PI & $49.99^{a}$ & $6.20^{a}$ & $4.20^{a}$ & $33.63^{a}$ & $7.62^{a}$ & $44.81^{b}$ & $17.49^{a}$ \\
\hline EE & 0.66 & 0.49 & 0.40 & 3.15 & 0.48 & 0.67 & 1.22 \\
\hline$P$ value & 0.001 & 0.444 & 0.634 & 0.600 & 0.716 & 0.001 & 0.555 \\
\hline \multicolumn{8}{|c|}{$\begin{array}{l}\mathrm{CON}=\text { dieta control; } \mathrm{Lb}=0.20 \mathrm{~g} \mathrm{~kg}^{-1} \text { Lippia berlandieri Schauer en dieta; } \mathrm{PI}=0.20 \mathrm{~g} \\
\mathrm{~kg}^{-1} \text { Poliomintha longiflora Gray en dieta; EE }=\text { error estándar. } \mathrm{L}^{*}=\text { Luminosidad; }{ }^{*}= \\
\text { tendencia a rojo; }{ }^{b *}=\text { tendencia al amarillo; Hue }=\text { ángulo Hue }(\text { tono); Chroma }=\text { índice de } \\
\text { saturación; } \Delta \mathrm{CT}=\text { cambio de color total; IC }=\text { índice de coloración. }{ }^{a-b} \text { Medias }(\mathrm{n}=8 \text { por } \\
\text { tratamiento) en columnas y con diferente superíndice son diferentes significativamente ( } \mathrm{P} \\
<0.05) \text {. }\end{array}$} \\
\hline
\end{tabular}

Tabla 6. Fuerza de corte y perfil de textura en pechugas de codornices suplementadas con aceites de orégano.

\begin{tabular}{|c|c|c|c|c|c|c|c|c|}
\hline \multirow{2}{*}{ Trat } & \multicolumn{8}{|c|}{ Variables de textura } \\
\hline & FC (gf) & Dur (gf) & Elast (mm) & Coh & Gom (g) & Mast (g mm) & Resist & $\operatorname{Adh}\left(\mathrm{g} \mathrm{s}^{-1}\right)$ \\
\hline CON & $634.20^{a}$ & $1259.80^{a}$ & $0.6584^{a}$ & $0.6588^{a}$ & $833.10^{a}$ & $552.70^{a}$ & $0.3537^{a}$ & $-2.09^{a}$ \\
\hline $\mathrm{Lb}$ & $671.80^{a}$ & $1137.60^{a}$ & $0.6274^{a}$ & $0.6191^{b}$ & $708.90^{a}$ & $448.20^{a}$ & $0.3235^{b}$ & $-2.06^{a}$ \\
\hline $\mathrm{PI}$ & $533.80^{a}$ & $1206.90^{a}$ & $0.6417^{a}$ & $0.6247^{b}$ & $761.30^{a}$ & $494.20^{a}$ & $0.3327^{b}$ & $-1.22^{a}$ \\
\hline EE & 45.63 & 71.77 & 0.01 & 0.01 & 51.37 & 31.93 & 0.01 & 0.38 \\
\hline$P$ value & 0.115 & 0.494 & 0.173 & 0.001 & 0.245 & 0.164 & 0.002 & 0.205 \\
\hline
\end{tabular}

Tabla 7. Evaluación sensorial afectiva de pechugas de pollos suplementadas con aceites de orégano.

\begin{tabular}{cccccc}
\hline Tratamientos & Color & Olor & Sabor & Suavidad & Aceptabilidad global \\
\hline CON & $4.80^{a}$ & $5.03^{a}$ & $4.87^{a}$ & $5.23^{a}$ & $4.80^{a}$ \\
Lb & $5.03^{a}$ & $5.23^{a}$ & $5.07^{a}$ & $5.43^{a}$ & $5.17^{a}$ \\
$\mathrm{PI}$ & $4.90^{a}$ & $5.37^{a}$ & $4.87^{a}$ & $5.00^{a}$ & $5.00^{a}$ \\
$\mathrm{EE}$ & 0.25 & 0.22 & 0.25 & 0.24 & 0.23 \\
$\mathrm{P}$ value & 0.477 & 0.389 & 0.773 & 0.320 & 0.434 \\
\hline
\end{tabular}

$\mathrm{CON}=$ dieta control; $\mathrm{Lb}=0.20 \mathrm{~g} \mathrm{~kg}^{-1}$ Lippia berlandieri Schauer en dieta; $\mathrm{PI}=$ $0.20 \mathrm{~g} \mathrm{~kg}^{-1}$ Poliomintha longiflora Gray en dieta; $\mathrm{EE}=$ error estándar. ${ }^{a}$ Medias ( $n=30$ consumidores) en columnas y con diferente superíndice son diferentes significativamente $(\mathrm{P}<0.05)$.

\section{LITERATURA CITADA}

Castillo LR, Portillo LJ, Leon FJ, Gutierrez DR, Angulo EM, Muy-Rangel MD, Heredia JB (2018) Inclusion of moringa leaf powder (Moringa oleifera) in fodder for feeding japanese quail (Coturnix coturnix japonica). Brazilian Journal of Poultry Science 20: 015-026.

Cázares-Gallegos R, Silva-Vázquez R, Hernández-Martínez CA, Gutiérrez-Soto JG, Kawas-Garza JR, Hume ME, Méndez-Zamora GM (2019) Performance, carcass variables, and meat quality in broilers supplemented with dietary Mexican oregano oil. Brazilian Journal of Poultry Science 21: 1-10.

Fatih Akdemir, Tahir Bayril, Sedat Baran M, Sener Yildiz A, Mucahit Kahraman, Cemal Orhan, Kazim Sahin (2018) The effect of dietary colostrum powder on performance, carcass yields and serum lipid peroxidation levels in Japanese quails (Coturnix coturnix japonica). Journal of Applied Animal Research 46 39-43.

Ghazaghi M, Mehri M, Bagherzadeh-Kasmani F (2014) Effects of dietary Mentha spicata on performance, blood 
metabolites, meat quality and microbial ecosystem of small intestine in growing Japanese quail. Animal Feed Science and Tecnology 194: 89-98.

Hajiaghapour M, Rezaeipour V (2018) Comparison of two herbal essential oils, probiotic, and mannan-oligosaccharides on egg production, hatchability, serum metabolites, intestinal morphology, and microbiota activity of quail breeders. Livestock Science 210: 93-98.

Hernández-Coronado AC, Silva-Vázquez R, Rangel-Nava ZE, Hernández-Martínez CA, Kawas-Garza JR, Hume ME, Méndez-Zamora G (2019) Mexican oregano essential oils given in drinking water on performance, carcass traits, and meat quality of broilers. Poultry Science 98: 3050-3058.

INEGI (2020) Instituto Nacional de Estadística y Geografía, México en Cifras. http://www3.inegi.org.mx/sistemas/ mexicocifras/default.aspx. Fecha de consulta: 28 de julio de 2020.

Lábaque MC, Kembro JM, Luna A, Marin RH (2013) Effects of thymol feed supplementation on female Japanese quail (Coturnix coturnix) behavioral fear response. Animal Feed Science and Technology 183: 67-72.

Mehdipour Z, Afsharmanesh M, Masoud S (2013) Effects of dietary synbiotic and cinnamon (Cinnamomum verum) supplementation on growth performance and meat quality in Japanese quail. Livestock Science 154: $152-157$.

Mehri M, Sabaghi V, Bagherzadeh-Kasmani F (2015) Mentha piperita (peppermint) in growing Japanese quails' diet: Serum biochemistry, meat quality, humoral immunity. Animal Feed Science and Tecnology 206: 57-66.

Méndez-Zamora G, García-Macías JA, Durán-Meléndez LA, Herman-Lara H, Santellano Estrada E, Silva-Vazquez R (2015a) Aceite esencial de orégano (Lippia berlandieri Schauer) en variables de calidad de la canal de pollo. Ecosistemas y Recursos Agropecuarios 2: 41-51.

Méndez-Zamora G, García-Macías JA, Santellano-Estrada E, Durán-Meléndez LA, Silva-Vazquez R (2015b) Aceite de orégano sobre la calidad de pechuga de pollos de engorda. Investigación y Ciencia 65: 5-12.

Minitabß (2014) User's Guide 2: Data Analysis and Quality Tools. Getting Started with Minitab 17. Version 17.3.0. Minitab, Inc. USA. https://shamsulsarip.files.wordpress.com/2015/07/minitab_book.pdf. Fecha de consulta: 3 de mayo de 2021.

Mnisi CM, Mlambo V (2018) Growth performance, haematology, serum biochemistry and meat quality characteristics of Japanese quail (Coturnix coturnix japonica) fed canola meal-based diets. Animal Nutrition 4: 37-43.

Naghmeh Dehghani, Mohsen Afsharmanesh, Mohammad Salarmoini, Hadi Ebrahimnejad, Ahmad Bitaraf (2018) Effect of pennyroyal, savory and thyme essential oils on Japanese quail physiology. Heliyon 4: e00881. DOI: 10.1016/j.heliyon.2018.e00881.

NRC (1994) Nutritional requirements of poultry. 9th ed. Natl. Acad. Sci. Washington, DC, USA. pp: 44-45.

Sadi Cetingul I, Ismail Bayram, Mehmet Yardimci, Hesna Sahin E, Erol Sengor, Burhaneddin-Akkaya A, CangirUyarlar (2009) Effects of oregano (Oregano Onites) on performance, hatchability and egg quality parameters of laying quails (Coturnix coturnix japonica). Italian Journal of Animal Science 8: 467-477.

Sánchez-Zamora N, Silva-Vázquez R, Rangel-Nava ZE, Hernández-Martínez CA, Kawas-Garza JR, Hume ME, Herrera-Balandrano DD, Méndez-Zamora G (2019) Inulina de agave y aceite de orégano mejoran la productividad de pollos de engorda. Ecosistemas y Recursos Agropecuarios 6: 523-534.

SE (2014) NOM-033-SAG/ZOO-2014 Norma Oficial Mexicana. Métodos para dar muerte a los animales domésticos y silvestres. http://www.economia-noms.gob.mx/noms/consultasAction.do. Fecha de consulta: 28 de julio de 2020 . 
Sugiharto S (2016) Role of nutraceuticals in gut health and growth performance of poultry. Journal of the Saudi Society of Agricultural Sciences 15: 99-111.

Vargas-Sanchez RD, Torrescano-Urrutia GR, Ibarra-Arias FJ, Portillo-Loera JJ, Rios-Rincon FG, Sanchez-Escalante A (2018) Effect of dietary supplementation with Pleurotus ostreatus on growth performance and meat quality of Japanese quail. Livestock Science 207: 117-125.

Yesilbag D, Cengiz SS, Cetin I, Meral Y, Biricik H (2014) Influence of Juniper (Juniperus communis) oil on growth performance and meat quality as a natural antioxidant in quail diets. British Poultry Science 55: 495-500.

Yesilbag D, Gezen SS, Biricik H, Bulbul T (2012) Effect of a rosemary and oregano volatile oil mixture on performance, lipid oxidation of meat and haematological parameters in Pharaoh quails. British Poultry Science 53: 89-97. 